\title{
Difference in Spinal Sagittal Alignment and Health- Related Quality of Life between Males and Females with Cervical Deformity
}

\author{
Shin Oe ${ }^{1}$, Daisuke Togawa ${ }^{1}$, Go Yoshida ${ }^{1}$, Tomohiko Hasegawa ${ }^{1}$, Yu Yamato ${ }^{1}$, Sho Kobayashi ${ }^{1}$, \\ Tatsuya Yasuda ${ }^{2}$, Tomohiro Banno ${ }^{1}$, Yuki Mihara ${ }^{1}$, Yukihiro Matsuyama ${ }^{1}$ \\ ${ }^{1}$ Department of Orthopedic Surgery, Hamamatsu University School of Medicine, Hamamatsu, Japan \\ ${ }^{2}$ Department of Orthopedic Surgery, Hamamatsu Medical Center, Hamamatsu, Japan
}

\section{Study Design: Large cohort study.}

Purpose: To clarify spinal sagittal alignment and health-related quality of life (HROOL) according to sex in volunteers aged $>50$ years with cervical deformity (CD).

Overview of Literature: Adult spinal deformities, especially those associated with lumbosacral lesions, are more frequent in females; however, $\mathrm{CD}$ is observed to a greater extent in males.

Methods: We divided 656 volunteers (263 males, 393 females; age, 50-89 years [mean, 73 years]) as follows: males with CD (CDM; 82 patients); males without CD (NCDM, 181); females with CD (CDF, 36); and females without CD (NCDF, 357). CD was defined as C2-7 sagittal vertical axis (SVA) $\geq 40 \mathrm{~mm}$. We measured pelvic tilt (PT), pelvic incidence minus lumbar lordosis (PI-LL), thoracic kyphosis, T1 slope, cervical lordosis, C7 SVA, and C2-7 SVA. HROOL was evaluated using Euro00L five dimensions questionnaire (EQ-5D). Results: In CDM, NCDM, CDF, and NCDF groups, the respective parameters were as follows: PT: $15^{\circ}, 14^{\circ}, 26^{\circ}$, and 21 $1^{\circ}$; PI-LL: $7^{\circ}$, $5^{\circ}, 16^{\circ}$, and 10 $0^{\circ}$ C2-7 SVA: 49, 24, 46, and $20 \mathrm{~mm}$; C7 SVA: 61, 40, 75, and $47 \mathrm{~mm}$; and EQ-5D: 0.82, 0.88, 0.78, and 0.81. PT and PILL were significantly greater in the CDF group than in the NCDF group $(p<0.05)$ but were not significantly different between CDM and NCDM groups. The CDF group already showed deterioration of spinopelvic alignment, although it was maintained in the CDM group. EQ-5D in showed significantly greater deterioration the CDM group than in the NCDM group; deterioration of lumbopelvic parameters had less influence in males $(p<0.05)$.

Conclusions: Sagittal spinal deformity may have different mechanisms in males and females. The deterioration of spinal sagittal alignment in males may originate from the cervical spine, and CD may be associated with HROOL.

Keywords: Cervical deformity; Cervical alignment, Health-related 00L; Spinal deformity; Sex differences

\section{Introduction}

It is generally known that spinal sagittal malalignment causes poor health-related quality of life (HRQOL) [1-5].
Because lumbar and pelvic parameters are strongly related to HRQOL, they have been well investigated. However, it has recently been reported that HRQOL deteriorates not only because of lumbar spine and pelvic malalignment but

Received Feb 9, 2017; Revised Mar 2, 2017; Accepted Mar 20, 2017

Corresponding author: Shin Oe

Department of Orthopedic Surgery, Hamamatsu University School of Medicine, 1-20-1 Handayama Higashi-ku, Hamamatsu, Shizuoka 431-3192, Japan

Tel: +81-53-435-2299; Fax: +81-53-435-2296, E-mail: mecersior@gmail.com 
also because of cervical deformity (CD) [6-11].

The prevalence of CD is high (53\%) in adults with spinal deformity [12]. However, it is unclear how CD impacts pelvic, lumbar, thoracic, and cervical alignments and HRQOL. Many reports have investigated spinal sagittal alignment, but few have investigated sex differences. We have previously reported that the deterioration of spinal deformity originates from the pelvis in females and the cervical spine in males [6]. Therefore, given that the mechanism underlying worsening of spinal sagittal alignment varies according to sex, it is important to evaluate the sex differences. We have also previously reported that CD is significantly associated with HRQOL and that deterioration starts after the age of 60 years [6]. Therefore, it is also important to evaluate CD in the elderly.

Considering these issues, we investigated sex differences in spinal sagittal alignment and HRQOL in volunteers with $C D$ aged $\geq 50$ years.

\section{Materials and Methods}

\section{Institutional Review Board}

The study protocol was approved by the Institutional Review Board of Hamamatsu University School of Medicine, Shizuoka, Japan (Toei Hospital; IRB No. 201201).

\section{Volunteer cohort}

We investigated 746 volunteers who participated in the Toei town musculoskeletal examination program in 2012. All volunteers were healthy who could walk to Toei Hospital alone without assistance. They joined our healthscreening program after receiving information from the general announcement by the Toei Hospital.

\section{Inclusion/exclusion criteria}

The inclusion criteria were age of 50-89 years, available whole-spine and pelvic X-rays taken with the patient in a standardized standing position, and informed consent provided. The exclusion criteria were Cobb angle of $\geq 25^{\circ}$ in the coronal plane; symptomatic osteoporotic fractures with severe wedge-shaped vertebra with Genant semiquantitative grade 3 [13]; history of other pathologies (tumor, infection, etc.), total joint arthroplasty, and instrumented spinal surgery; and unable to stand without assistance.

\section{Radiological assessment}

The radiographic technique was standardized as follows: (1) the volunteers were in a standing position, looking straight ahead with the hands placed on the clavicle; (2) acquisition of three views (anteroposterior and lateral craniopelvic views and lateral pelvic view); and (3) a distance of $1.5 \mathrm{~m}$ between the $\mathrm{X}$-ray tube and radiograph. The digitized radiographs were transferred as Digital Imaging and Communications in Medicine data to a computer. Radiographic parameters were measured using imaging software (Surgimap Spine, Nemaris, Inc., New York, USA). Radiological measurements were reviewed by two experienced spine surgeons.

\section{Measured parameters}

The measured radiographic parameters were as follows: (1) pelvic tilt (PT), (2) sacral slope (SS), (3) pelvic incidence (PI), (4) pelvic incidence minus lumbar lordosis (PI-LL), (5) LL (the angle between the L1 and S1 superior endplates), (6) thoracic kyphosis (TK; the angle between the T5 superior and T12 inferior endplates), (7) T1 slope (T1S; the angle between the horizontal plane and T1 superior endplate), (8) cervical lordosis (CL; the angle between the C2 and C7 inferior endplates), (9) C7 sagittal vertical axis (SVA; the distance between a plumb line from the center of the C7 vertebral body and posterior superior corner of the sacrum), (10) C2 SVA (the distance between a plumb line from the center of the $\mathrm{C} 2$ vertebral body and posterior superior corner of the sacrum), (11) C2-7 SVA (the distance between a plumb line from the center of the $\mathrm{C} 2$ vertebral body and posterior superior corner of C7), and (12) T1S-CL.

\section{Clinical evaluation}

The EuroQOL five dimensions questionnaire (EQ-5D) short-form health survey was used to assess HRQOL [14]. EQ-5D is a scale consisting of five dimensions (mobility, self-care, usual activities, pain/discomfort, and anxiety/ depression). A score of $0-1$ indicates that one is perfectly healthy and a lower score indicates an unhealthy state.

\section{Statistical analysis}

SPSS vers. 21(IBM-SPSS, Inc., Chicago, IL, USA) was used for statistical analyses. Unpaired $t$-tests were used for 
analysis of sex differences. Analysis of variance and the Tukey's test (for post hoc analysis) were used for multiple comparisons. Spearman's rank correlation coefficient was analyzed for the correlation of each parameter with EQ5D. A $p<0.05$ was considered statistically significant.

\section{Definition of $\mathrm{CD}$ and grouping}

According to previous reports, $\mathrm{CD}$ was defined as $\mathrm{C} 2-7$ SVA $>40 \mathrm{~mm}[6,12,15-17]$. The volunteers were divided into four groups: males with $\mathrm{CD}(\mathrm{CDM})$, males without $\mathrm{CD}(\mathrm{NCDM})$, females with $\mathrm{CD}(\mathrm{CDF})$, and females without $\mathrm{CD}(\mathrm{NCDF})$.

\section{Results}

\section{Demographics}

Among the 746 volunteers considered for participation in this study, 90 were excluded because they could not be assessed using the radiographs or questionnaire for HRQOL. Thus, 656 were included for analysis (mean age, $72.7 \pm 7.9$ years; range, $50-89$ years), with 82 (mean age, $75.4 \pm 6.1$ years), 181 (mean age, $71.9 \pm 8.2$ years), 36 (mean age, $76.8 \pm 7.7$ years), and 357 (mean age, $72.0 \pm 7.5$ years) in the CDM, NCDM, CDF, and NCDF groups, respectively (Table 1). The volunteers in the CDM and CDF groups were significantly older than those in the NCDM and NCDF groups $(p<0.01)$. There were no differences in the body mass index in the groups. The intraclass correlation coefficients for intra/interobserver reliabilities were 0.967/0.969, 0.995/0.996, 0.996/0.990, and 0.918/0.966 for C2-7 SVA, C7 SVA, PT, and PI, respectively [18].

\section{Pelvic and lumbar parameters}

As shown in Table 2, there were no significant differences in the mean PT between the CDM $\left(15^{\circ}\right)$ and $\operatorname{NCDM}\left(14^{\circ}\right)$ groups. On the other hand, $\mathrm{PT}\left(26^{\circ}\right)$ in the $\mathrm{CDF}$ group was significantly higher than that $\left(21^{\circ}\right)$ in the NCDF group $(p<0.05)$. PT in females tended to be greater than that in males regardless of $\mathrm{CD}$.

The mean SS $\left(29^{\circ}\right)$ in the CDM group was significantly lower than that $\left(32^{\circ}\right)$ in the NCDM group $(p<0.05)$. There was no significant difference in SS between the CDF $\left(28^{\circ}\right)$ and NCDF $\left(30^{\circ}\right)$ groups. The results for PI were similar

Table 1. Demographic data for study population

\begin{tabular}{lcccccc} 
Group & CDM & NCDM & CDF & NCDF & $p($ ANOVA) & $p(p o s t ~ h o c)$ \\
No. & 82 & 181 & 36 & 357 & - & - \\
Age $(\mathrm{yr})$ & $75.4 \pm 6.1$ & $71.9 \pm 8.2$ & $76.8 \pm 7.7$ & $72.0 \pm 7.5$ & $<0.001^{* * *}$ & $* * * \mathrm{a}, \mathrm{d}, f^{* * *}: \mathrm{C}$ \\
Body mass index $\left(\mathrm{kg} / \mathrm{m}^{2}\right)$ & $23.3 \pm 2.7$ & $22.5 \pm 2.7$ & $22.5 \pm 3.2$ & $22.4 \pm 3.0$ & 0.097 & - \\
\hline
\end{tabular}

CDM, males with cervical deformity; NCDM, males without cervical deformity; CDF, females with cervical deformity; NCDF, females without cervical deformity; ANOVA, analysis of variance.

a, CDM vs. NCDM; b, CDM vs. CDF; c, CDM vs. NCDF; d, NCDM vs. CDF; e, NCDM vs. NCDF; f, CDF vs. NCDF.

${ }^{*} p<0.05$; ${ }^{* *} p<0.01 ;{ }^{* * *} p<0.001$.

Table 2. Pelvis and lumbar parameters

\begin{tabular}{|c|c|c|c|c|c|c|}
\hline Group & CDM & NCDM & CDF & NCDF & $p$ (ANOVA) & $p$ (post hoc) \\
\hline No. & 82 & 181 & 36 & 357 & & \\
\hline PT & $15.3 \pm 8.9$ & $14.3 \pm 7.4$ & $26.2 \pm 9.8$ & $21.4 \pm 10.1$ & $<0.001^{* * *}$ & ${ }^{*}: f,{ }^{* *}: b, c, d, e$ \\
\hline SS & $29.0 \pm 9.0$ & $32.3 \pm 8.7$ & $27.5 \pm 10.5$ & $29.5 \pm 11.0$ & $0.005^{* *}$ & ${ }^{*}: a^{*}{ }^{*}: \mathrm{e}$ \\
\hline $\mathrm{PI}$ & $44.3 \pm 9.4$ & $46.6 \pm 9.3$ & $53.7 \pm 11.7$ & $50.3 \pm 12.5$ & $<0.001^{* * *}$ & ${ }^{* *}: d, e,{ }^{* * *}: b, c$ \\
\hline $\mathrm{LL}$ & $37.5 \pm 15.0$ & $41.7 \pm 13.0$ & $37.8 \pm 18.5$ & $40.4 \pm 16.3$ & 0.171 & - \\
\hline PI-LL & $6.8 \pm 15.9$ & $4.9 \pm 12.8$ & $15.9 \pm 21.5$ & $9.9 \pm 16.9$ & $<0.001^{* * *}$ & ${ }^{*}: d_{1}{ }^{* *}: e$ \\
\hline
\end{tabular}

CDM, males with cervical deformity; NCDM, males without cervical deformity; CDF, females with cervical deformity; NCDF, females without cervical deformity; ANOVA, analysis of variance; PT, pelvic tilt; SS, sacral slope; PI, pelvic incidence; LL, lumbar lordosis.

a, CDM vs. NCDM; b, CDM vs. CDF; c, CDM vs. NCDF; d, NCDM vs. CDF; e, NCDM vs. NCDF; f, CDF vs. NCDF.

${ }^{*} p<0.05 ;{ }^{* *} p<0.01 ;{ }^{* *} p<0.001$. 
to those for PT because PI in females tended to be higher than that in males regardless of CD. The mean LLs in the $\mathrm{CDM}, \mathrm{NCDM}, \mathrm{CDF}$, and $\mathrm{NCDF}$ groups were $38^{\circ}, 42^{\circ}, 38^{\circ}$, and $40^{\circ}$, respectively. There was no significant difference among all groups, although the mean LL in volunteers with $\mathrm{CD}$ tended to be lower. Moreover, there was no discrepancy in LL in terms of sex.

PI-LL in females also tended to be higher than that in males regardless of CD. Furthermore, PI-LL $\left(16^{\circ}\right)$ in the $\mathrm{CDF}$ group was higher than that $\left(10^{\circ}\right)$ in the NCDF group, although the difference was not statistically significant $(p=0.146)$. On the other hand, the difference in the mean PI-LL between the CDM $\left(7^{\circ}\right)$ and $\mathrm{NCDM}\left(5^{\circ}\right)$ groups was small and not significant $(p=0.813)$.

These results suggested that females with $C D$ also had deteriorated pelvic alignment, whereas males with $\mathrm{CD}$ had no pelvic malalignment.

\section{Cervicothoracic parameters}

As shown in Table 3 , the mean TK $\left(45^{\circ}\right)$ in the CDF group was significantly higher than that $\left(35^{\circ}\right)$ in the NCDF group $(p<0.05)$ and tended to be higher than that $\left(37^{\circ}\right)$ in the CDM group, although there was no significant difference. The mean TK in the CDF group was the highest among all groups.

The mean T1S was significantly higher in the groups with $\mathrm{CD}$ than in those without $\mathrm{CD}(p<0.001)$. Moreover, the mean T1S in the CDF group $\left(39^{\circ}\right)$ tended to be higher than that in the CDM group $\left(37^{\circ}\right)$, although there was no significant difference. The mean T1S in the CDF group was the highest among all groups, similar to the result for TK.

The mean CL in the groups with CD tended to be lower. In particular, the mean $\mathrm{CL}\left(8^{\circ}\right)$ in the $\mathrm{CDM}$ group was significantly lower than that $\left(14^{\circ}\right)$ in the NCDM group.

The mean T1S-CL was the highest in the CDM group $\left(29^{\circ}\right)$. In the CDF group $\left(28^{\circ}\right)$, the mean T1S-CL was also $>20^{\circ}$, which was one of the definitions for $\mathrm{CD}$ provided by Ames et al. [16] and Passias et al. [17].

The mean C2-7 SVA in the CDM, NCDM, CDF, and NCDF groups was 49, 24, 46, $20 \mathrm{~mm}$, respectively. C2-7 SVA in the CDM group was the most severely deteriorated parameter among all groups. These results suggested that $\mathrm{CD}$ was more severe in males compared with females.

Table 3. Cervicothoracic parameters

\begin{tabular}{|c|c|c|c|c|c|c|}
\hline Group & CDM & NCDM & $\mathrm{CDF}$ & NCDF & $p(\mathrm{ANOVA})$ & $p$ (post hoc) \\
\hline No. & 82 & 181 & 36 & 357 & & \\
\hline TK & $36.6 \pm 12.6$ & $33.6 \pm 11.2$ & $45.1 \pm 17.5$ & $35.2 \pm 14.6$ & $<0.001^{* * *}$ & ${ }^{*}: f_{,}^{* *}: d$ \\
\hline T1S & $37.1 \pm 6.9$ & $31.4 \pm 5.8$ & $39.3 \pm 8.8$ & $31.0 \pm 7.7$ & $<0.001^{* * *}$ & ${ }^{* * *}: a, c, d, f$ \\
\hline $\mathrm{CL}$ & $8.0 \pm 7.0$ & $14.2 \pm 8.8$ & $10.8 \pm 9.2$ & $14.9 \pm 10.4$ & $<0.001^{* * *}$ & ${ }^{* * *}: a, c$ \\
\hline T1S-CL & $29.1 \pm 6.7$ & $17.5 \pm 8.1$ & $28.4 \pm 6.6$ & $16.8 \pm 7.9$ & $<0.001^{* * *}$ & $* * *: a, c, d, f$ \\
\hline C2-7 SVA & $49.1 \pm 8.8$ & $24.3 \pm 9.2$ & $46.2 \pm 4.7$ & $20.0 \pm 9.6$ & $<0.001^{* * *}$ & $* * *: a, c, d, e, f$ \\
\hline
\end{tabular}

CDM, males with cervical deformity; NCDM, males without cervical deformity; CDF, females with cervical deformity; NCDF, females without cervical deformity; ANOVA, analysis of variance; TK, thoracic kyphosis; T1S, T1 slope; CL, cervical lordosis; C2-7 SVA, C2-7 sagittal vertical axis. a, CDM vs. NCDM; b, CDM vs. CDF; c, CDM vs. NCDF; d, NCDM vs. CDF; e, NCDM vs. NCDF; f, CDF vs. NCDF. ${ }^{*} p<0.05 ;{ }^{* *} p<0.01 ;{ }^{* * *} p<0.001$.

Table 4. C7 SVA, C2 SVA, and EQ-5D

\begin{tabular}{|c|c|c|c|c|c|c|}
\hline Group & CDM & NCDM & CDF & NCDF & $p$ (ANOVA) & $p$ (post hoc) \\
\hline No. & 82 & 181 & 36 & 357 & - & - \\
\hline C7 SVA & $61.3 \pm 48.3$ & $40.3 \pm 38.6$ & $75.2 \pm 64.3$ & $46.8 \pm 45.5$ & $<0.001^{* * *}$ & ${ }^{*}: d^{* * *}: a$ \\
\hline C2 SVA & $110.5 \pm 52.0$ & $66.2 \pm 38.7$ & $124.1 \pm 58.4$ & $67.6 \pm 45.8$ & $<0.001^{* * *}$ & ${ }^{* * *}: a, c, d, f$ \\
\hline EQ-5D & $0.815 \pm 0.162$ & $0.878 \pm 0.152$ & $0.777 \pm 0.174$ & $0.814 \pm 0.162$ & $<0.001^{* * *}$ & ${ }^{*}: \mathrm{a}^{* *}: \mathrm{d}^{* * *}: \mathrm{e}$ \\
\hline
\end{tabular}

SVA, sagittal vertical axis; EQ-5D, EuroQ0L-5 dimension; CDM, males with cervical deformity; NCDM, males without cervical deformity; CDF, females with cervical deformity; NCDF, females without cervical deformity; ANOVA, analysis of variance. 


\section{Parameters of C7 SVA, C2 SVA, and EQ-5D}

C7 SVA in the CDM, NCDM, CDF, and NCDF groups was $61,40,75$, and $47 \mathrm{~mm}$, respectively (Table 4), and tended to be higher in the groups with $\mathrm{CD}$ than in those without $\mathrm{CD}$. The difference was significant between the CDM and NCDM groups $(p<0.01)$ but not between the $\mathrm{CDF}$ and NCDF groups $(p=0.063)$.

C2 SVA in the four groups was 111, 66, 124, and $68 \mathrm{~mm}$, respectively, and was significantly higher in the groups with $\mathrm{CD}$ than in those without $\mathrm{CD}(p<0.001)$. However, there was no significant difference between males and females with $\mathrm{CD}$.

EQ-5D in the four groups was $0.815,0.878,0.777$, and 0.814 , respectively. The mean EQ-5D in the CDF group was the poorest among all groups. On the other hand, EQ-5D was significantly lower in the CDM than in the NCDM groups $(p<0.05)$.

\section{Relation between EQ-5D and each parameter}

As shown in Table 5, the parameters in females for which EQ-5D had a weak correlation were PT ( $r=-0.239$, $p=0.001)$, PI-LL $(r=-0.252, p=0.001)$, C7 SVA $(r=-0.346$, $p=0.001)$, and C2 SVA $(r=-0.344, p=0.001)$. These were lumbopelvic and global parameters. On the other hand, the parameters in males for which EQ-5D had correlation were not observed. In other words, the mechanism of deteriorated EQ-5D was different between males and females.

\section{Discussion}

Few large-scale studies have evaluated cervical sagittal alignment $[6,19]$. Our results indicated that the mechanism underlying spinal deformity in spinal sagittal alignment was different between males and females. As shown in Fig. 1, the CDF group already had malalignment of the pelvis and thoracic spine compared with the NCDF group. On the other hand, there was no significant difference in pelvic and thoracic parameters between the CDM and NCDM groups (Fig. 2). Moreover, Fig. 3 showed that only the pelvic parameters were different between the CDM and CDF groups. Ames et al. [20] reported a correlation with adjacent sagittal parameters. Therefore, CD can initially impact the thoracic spine and subsequently result in deterioration of the lumbar spine and pelvis. Our results indicated that the deterioration of sagittal alignment originated from the cervical spine in males because pelvic alignment in the CDM group was maintained, whereas

Table 5. Spearman's rank correlation coefficient between EQ-5D and each parameter

\begin{tabular}{|c|c|c|c|c|c|c|}
\hline \multirow{2}{*}{ Group } & \multicolumn{2}{|c|}{ Total (no. $=656$ ) } & \multicolumn{2}{|c|}{ Female (no.=393) } & \multicolumn{2}{|c|}{ Male (no.=263) } \\
\hline & $r$ & $p$ & $r$ & $p$ & $r$ & $p$ \\
\hline PT & -0.216 & $<0.001^{* * *}$ & -0.239 & $<0.001^{* * *}$ & -0.108 & 0.082 \\
\hline SS & 0.088 & $0.024^{*}$ & 0.074 & 0.145 & 0.077 & 0.215 \\
\hline $\mathrm{Pl}$ & -0.127 & $0.001^{* *}$ & -0.147 & $0.004^{* *}$ & -0.032 & 0.607 \\
\hline LL & 0.123 & $0.002^{* *}$ & 0.164 & $0.001^{* *}$ & 0.063 & 0.307 \\
\hline PI-LL & -0.206 & $<0.001^{* * *}$ & -0.252 & $<0.001^{* * *}$ & -0.098 & 0.114 \\
\hline TK & -0.044 & 0.256 & -0.013 & 0.797 & -0.082 & 0.185 \\
\hline T1S & -0.075 & 0.056 & -0.148 & $0.003^{* *}$ & 0.011 & 0.857 \\
\hline $\mathrm{CL}$ & 0.026 & 0.509 & -0.044 & 0.380 & 0.170 & $0.006^{* *}$ \\
\hline T1S-CL & -0.076 & 0.051 & -0.092 & 0.068 & -0.120 & 0.051 \\
\hline C2-7 SVA & -0.021 & 0.596 & -0.036 & 0.477 & -0.148 & $0.016^{*}$ \\
\hline C7 SVA & -0.265 & $<0.001^{* * *}$ & -0.346 & $<0.001^{* * *}$ & -0.141 & $0.022^{*}$ \\
\hline C2 SVA & -0.262 & $<0.001^{* * *}$ & -0.344 & $<0.001^{* * *}$ & -0.179 & $0.004^{* *}$ \\
\hline
\end{tabular}

EQ-5D, Euro00L-5 dimension; $r$, correlation coefficient; PT, pelvic tilt; SS, sacral slope; PI, pelvic incidence; LL, lumbar lordosis; TK, thoracic kyphosis; T1S, T1 slope; CL, cervical lordosis; SVA, sagittal vertical axis.

${ }^{*} p<0.05 ;{ }^{* *} p<0.01 ;{ }^{* * *} p<0.001$. 

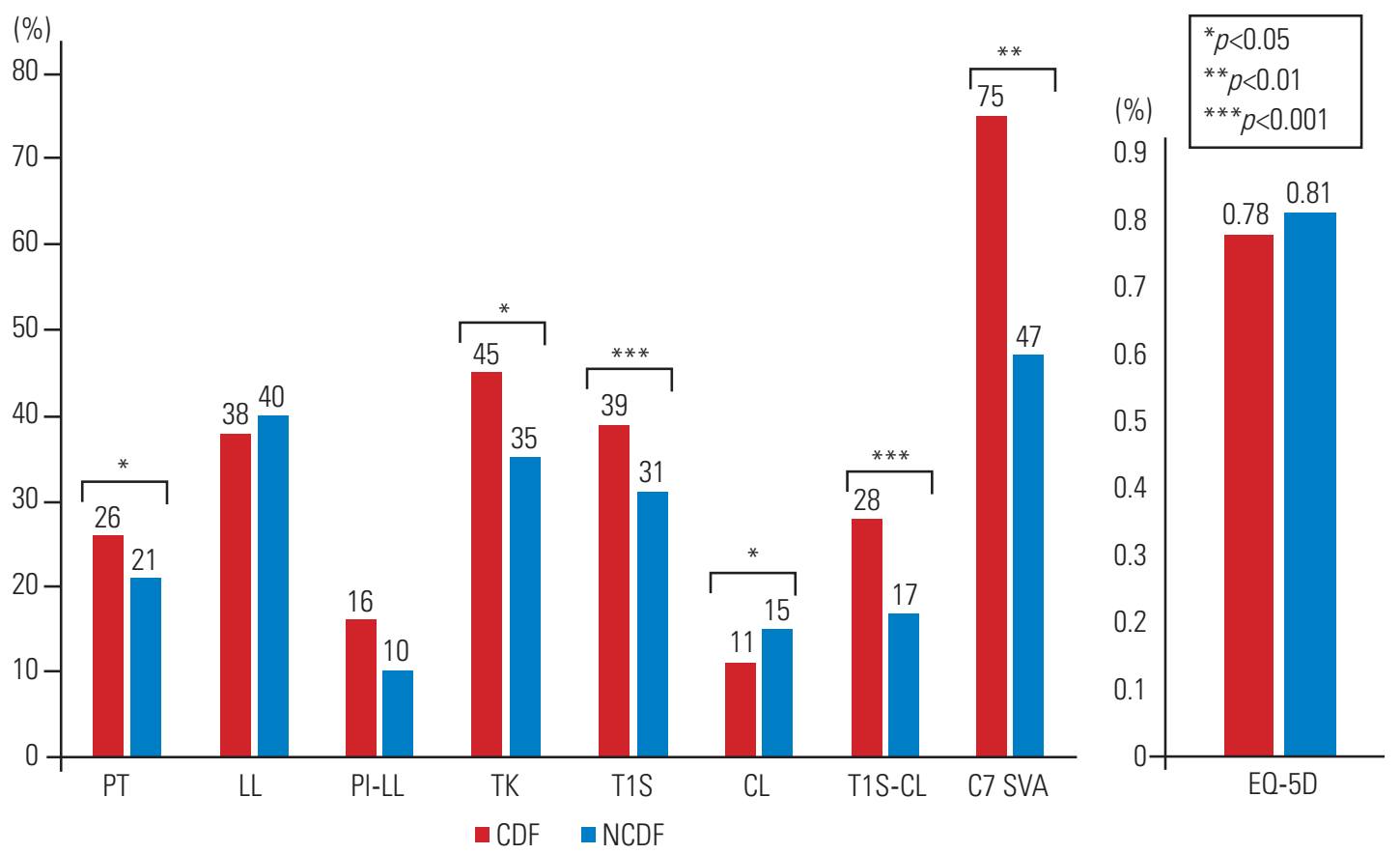

Fig. 1. Comparison between females with cervical deformity (CDF) and females without cervical deformity (NCDF). The parameters in the CDF group that significantly deteriorated were pelvic tilt, cervical parameters, and C7 SVA ( $p<0.05)$. PT, pelvic tilt; LL, lumbar lordosis; PI, pelvic incidence; TK, thoracic kyphosis; T1S, T1 slope; CL, cervical lordosis; SVA, sagittal vertical axis; EQ$5 \mathrm{D}$, using Euro00L five dimensions questionnaire.

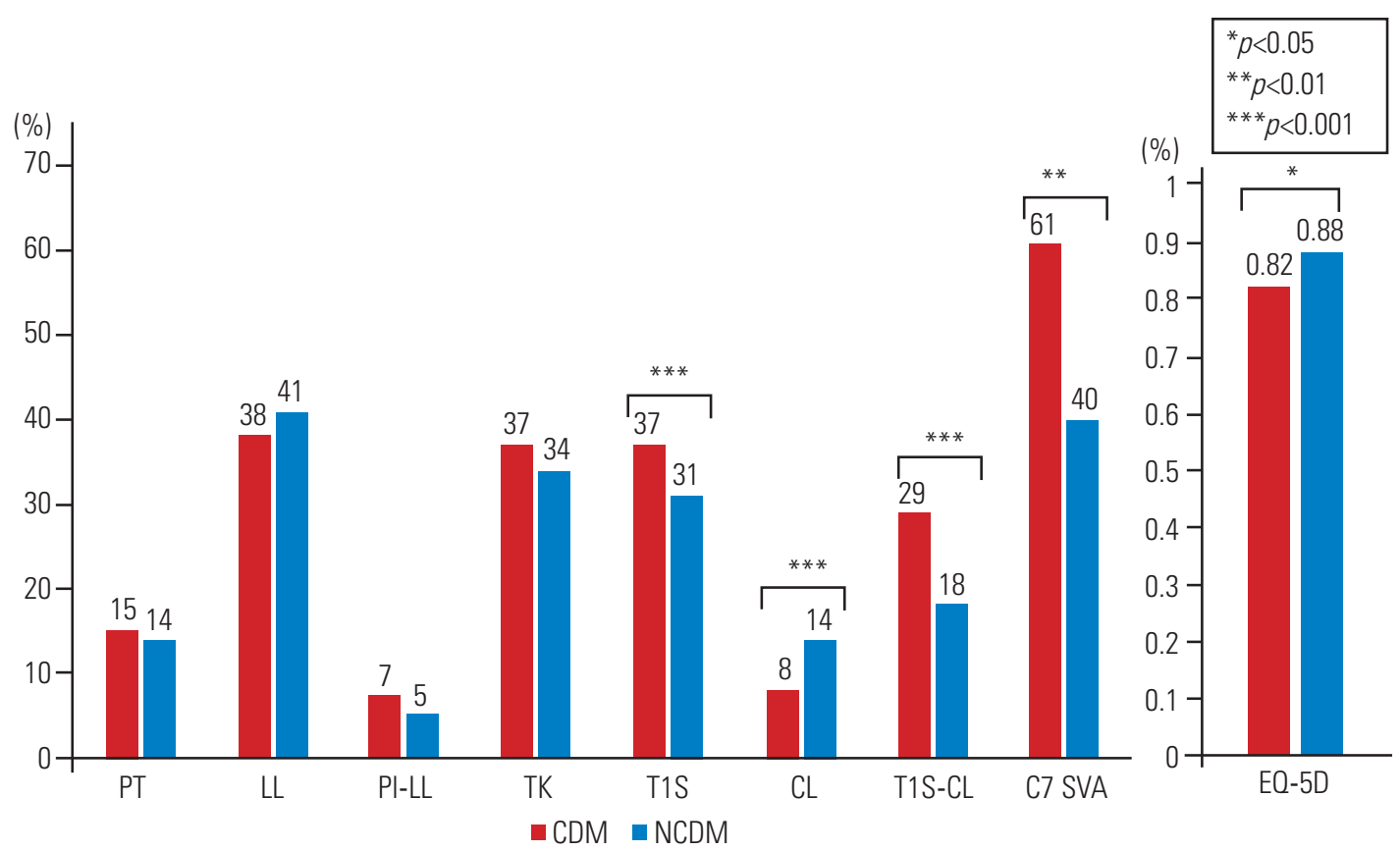

Fig. 2. Comparison between males with cervical deformity (CDM) and males without cervical deformity (NCDM). There were no significant differences in spinal parameters except for cervical parameters and C7 SVA. However, EuroQOL five dimension questionnaire (EQ-5D) score was significantly poorer in the CDM compared with the NCDM groups $(p<0.05)$. PT, pelvic tilt; LL, lumbar lordosis; PI, pelvic incidence; TK, thoracic kyphosis; T1S, T1 slope; CL, cervical lordosis; SVA, sagittal vertical axis.

in females, the deformity originated from the pelvis because the CDF group had not only cervical malalignment but also pelvic malalignment and the pelvic parameters in the NCDF group were significantly deteriorated than those in the NCDM group (Fig. 4). In fact, CD occurred more frequently in males $(31.1 \%)$ than in females $(10.1 \%)$. 

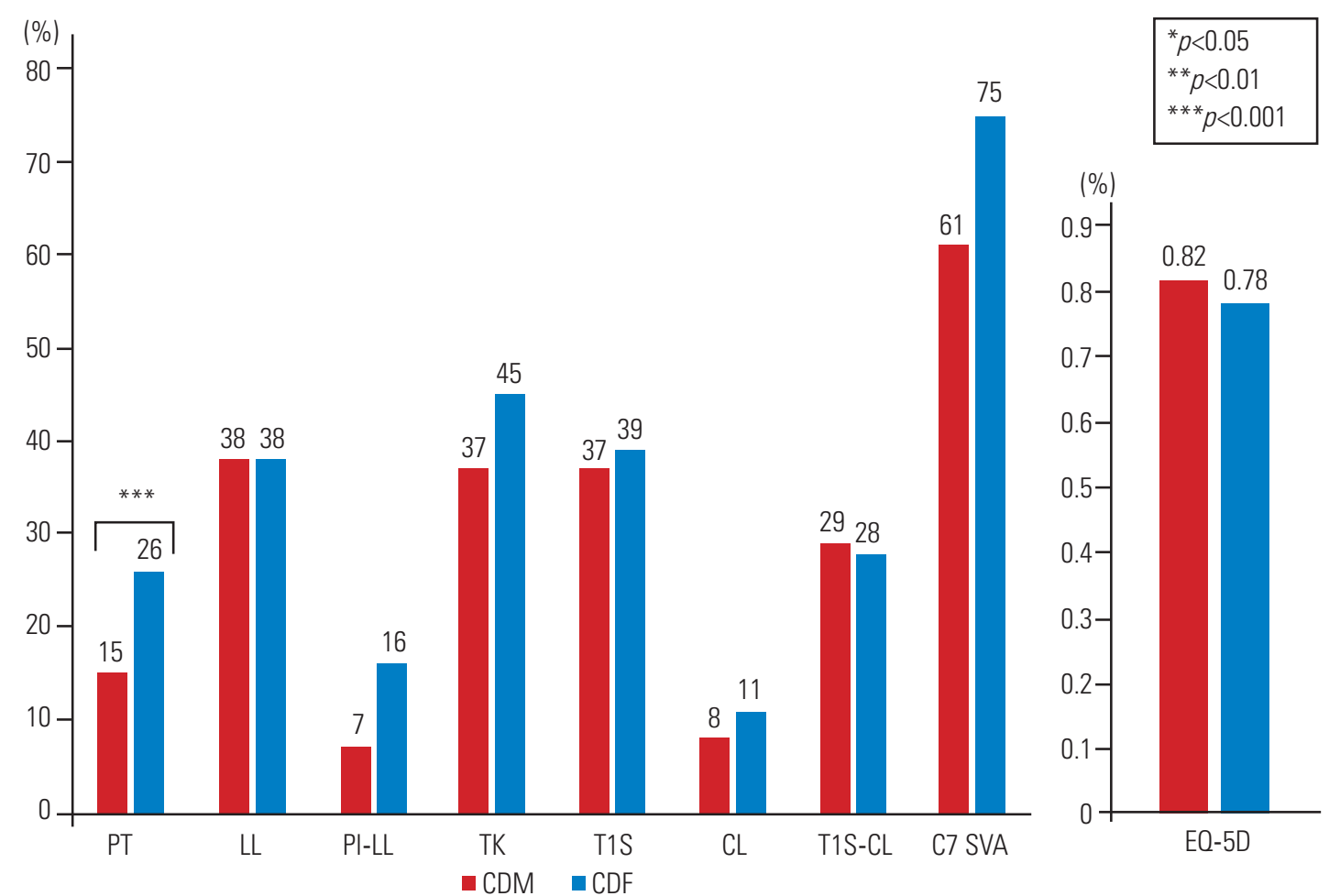

Fig. 3. Comparison between males with cervical deformity (CDM) and females with cervical deformity (CDF). PT significantly deteriorated in the CDF group than in the CDM group $(p<0.001)$, although no significant difference was noted for cervical parameters and C7 SVA. PT, pelvic tilt; LL, lumbar lordosis; PI, pelvic incidence; TK, thoracic kyphosis; T1S, T1 slope; CL, cervical lordosis; SVA, sagittal vertical axis; EQ-5D, Euro quality of life 5-dimension.

However, why spinal deformity originates from the pelvis in females and cervical spine in males remains unclear. Further research related to this issue is warranted.

However, the difference in LL between males and females was not observed (Table 2). Siemionow et al. [21] also reported that lumbar disc degeneration similarly develops between males and females. This result also suggests that kyphotic changes in the lumbar spine have no difference in terms of sex because a decrease in LL is related to lumbar disc degeneration.

Yukawa et al. [22] reported that CL increases with age. However, our study showed the opposite result, that is, that $\mathrm{CL}$ in the CDM and CDF (older) groups was lower than in the NCDM and NCDF (younger) groups. An increase in CL usually occurred as a result of compensation to maintain the patients' horizontal gaze in case TK or T1S was deteriorated because of lumbopelvic deformity. In other words, this result suggested that it is possible for volunteers with $\mathrm{CD}$ to have a decreased CL because this compensation mechanism would already have collapsed.

Several reports have shown that C2-7 SVA $>40 \mathrm{~mm}$ is significantly correlated with HRQOL $[6,7,23]$. However, none of these studies excluded the influence of deteriorated lumbopelvic alignment. In our study, no obvious difference in lumbopelvic alignment was observed between the CDM and NCDM groups. Therefore, the influence of lumbopelvic alignment was not associated with the EQ$5 \mathrm{D}$ score in males. This study verified that $\mathrm{CD}$ was significantly associated with HRQOL because EQ-5D in the CDM group (0.815) was significantly deteriorated compared with that in the NCDM group (0.878). To the best of our knowledge, this was the first study in which $\mathrm{CD}$ showed a significant correlation with HRQOL without the influence of lumbopelvic alignment.

Moreover, almost all correlative parameters with EQ5D were lumbar and pelvic parameters (PT and PI-LL) in females except for C2 SVA and C7 SVA. However, these parameters in males were not observed. Therefore, although it is usually considered that increased C7 SVA due to lumbopelvic deformity is correlated with HRQOL, our findings suggested that the main cause of deterioration of HRQOL in males is unclear. Some reports have also shown that sagittal spinal deformities in males deteriorated from cervical segments [24,25]. Moreover, some 


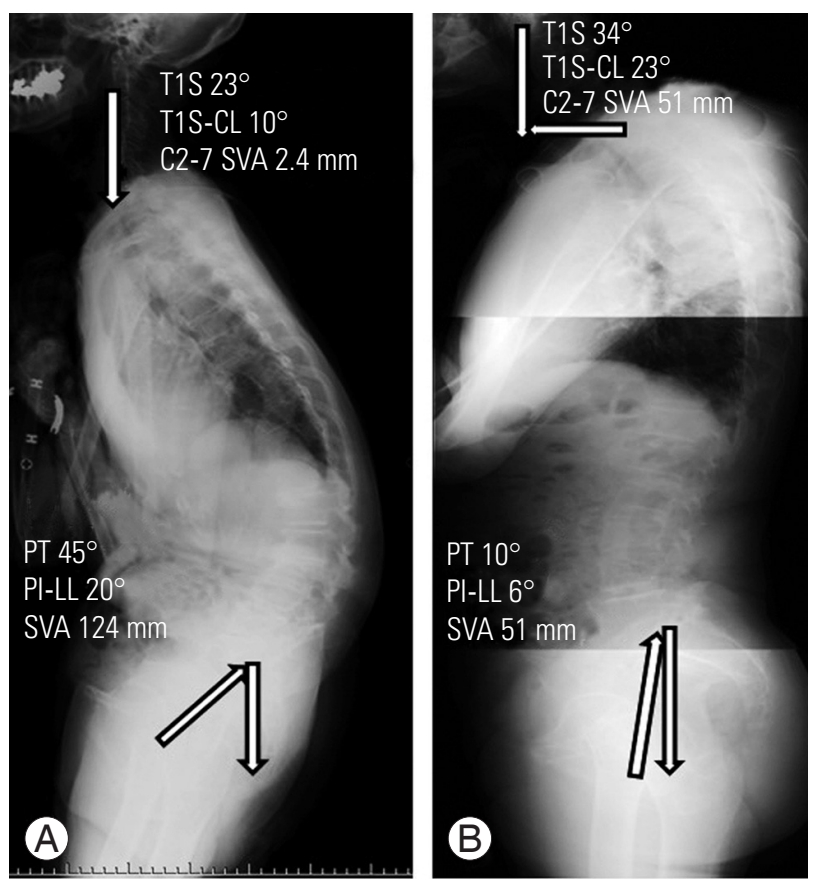

Fig. 4. Case presentations. (A) A 77-year-old female had a severe pelvic deformity (PT, 46 ${ }^{\circ}$; PI-LL, 20'; and C7 SVA, $124 \mathrm{~mm}$ ), while her cervical parameters were maintained (T1S, 23; T1S-CL, 10 ; and C2-7 SVA, $2.4 \mathrm{~mm}$ ). The spinal deformity originated from the lumbopelvic region in this case. (B) The pelvic parameters were almost in the normal range in a 73-year-old male (PT, 10 $\mathrm{PI}-\mathrm{LL}, 6^{\circ}$; and $\mathrm{C7}$ SVA, $51 \mathrm{~mm}$ ). However, he had a cervical deformity (T1S, 34'; T1S$\mathrm{CL}, 23^{\circ}$; and $\left.\mathrm{C} 2-7 \mathrm{SVA}, 51 \mathrm{~mm}\right)$. The spinal deformity originated from the cervical region in this case. PT, pelvic tilt; PI, pelvic incidence; LL, lumbar lordosis; SVA, sagittal vertical axis; T1S, T1 slope; CL, cervical lordosis.

reports have suggested that a high C2-7 SVA is strongly associated with cervical spondylotic myelopathy because of an increase in intramedullary pressure [9-11,19,26]. Therefore, when males with lumbopelvic deformity are treated, attention should also be paid to cervical alignment.

The primary limitation of this study was that all enrolled volunteers were Japanese and were aged $>50$ years. Therefore, it is unclear whether these data can be applied to other races. Second, almost all volunteers who participated in this study lived in mountainous settlements. Therefore, the environment was different from that of urban dwellers. The third limitation was the HRQOL assessment technique. Evaluation for CD may be suitable for tests, such as the Neck Disability Index questionnaire [27]. However, there have been no questionnaires specific to CD till date [22]. The fourth limitation was that the population between males and females was quite different. Finally, the number of volunteers in the CDF group was lower, which might explain why the EQ-5D score in the CDF group showed no significant difference from that in the CDM or NCDF groups, regardless of the CDF group having the poorest score among all groups.

\section{Conclusions}

Our study demonstrated that spinal deformity originates from lumbopelvic lesions in females and from cervical lesions in males. Moreover, CD may be significantly associated with HRQOL.

\section{Conflict of Interest}

Shin Oe and Daisuke Togawa belong to donated fund laboratory called Division of Geriatric Musculoskeletal Health.

\section{Acknowledgments}

Source of funding as bellows: Medtronic Sofamor Danek Inc., Japan Medical Dynamic Marketing Inc., Meitoku Medical Institution Jyuzen Memorial Hospital.

We have not received funding from the NIH, HHMI, or others.

\section{References}

1. Glassman SD, Bridwell K, Dimar JR, Horton W, Berven $S$, Schwab F. The impact of positive sagittal balance in adult spinal deformity. Spine (Phila Pa 1976) 2005;30:2024-9.

2. Glassman SD, Berven S, Bridwell K, Horton W, Dimar JR. Correlation of radiographic parameters and clinical symptoms in adult scoliosis. Spine (Phila Pa 1976) 2005;30:682-8.

3. Schwab FJ, Smith VA, Biserni M, Gamez L, Farcy JP, Pagala M. Adult scoliosis: a quantitative radiographic and clinical analysis. Spine (Phila Pa 1976) 2002;27:387-92.

4. Schwab F, Farcy JP, Bridwell K, et al. A clinical impact classification of scoliosis in the adult. Spine (Phila Pa 1976) 2006;31:2109-14.

5. Lafage V, Schwab F, Patel A, Hawkinson N, Farcy JP. Pelvic tilt and truncal inclination: two key radiographic parameters in the setting of adults with spinal deformity. Spine (Phila Pa 1976) 2009;34:E599-606. 
6. Oe S, Togawa D, Nakai K, et al. The influence of age and sex on cervical spinal alignment among volunteers aged over 50. Spine (Phila Pa 1976) 2015;40:1487-94.

7. Tang JA, Scheer JK, Smith JS, et al. The impact of standing regional cervical sagittal alignment on outcomes in posterior cervical fusion surgery. Neurosurgery 2012;71:662-9.

8. Chavanne A, Pettigrew DB, Holtz JR, Dollin N, Kuntz Ct. Spinal cord intramedullary pressure in cervical kyphotic deformity: a cadaveric study. Spine (Phila Pa 1976) 2011;36:1619-26.

9. Shamji MF, Ames CP, Smith JS, Rhee JM, Chapman JR, Fehlings MG. Myelopathy and spinal deformity: relevance of spinal alignment in planning surgical intervention for degenerative cervical myelopathy. Spine (Phila Pa 1976) 2013;38(22 Suppl 1):S147-8.

10. Smith JS, Lafage V, Ryan DJ, et al. Association of myelopathy scores with cervical sagittal balance and normalized spinal cord volume: analysis of 56 preoperative cases from the AOSpine North America Myelopathy study. Spine (Phila Pa 1976) 2013;38(22 Suppl 1):S161-70.

11. Fehlings MG, Tetreault LA, Wilson JR, Skelly AC. Cervical spondylotic myelopathy: current state of the art and future directions. Spine (Phila Pa 1976) 2013;38(22 Suppl 1):S1-8.

12. Smith JS, Lafage V, Schwab FJ, et al. Prevalence and type of cervical deformity among 470 adults with thoracolumbar deformity. Spine (Phila Pa 1976) 2014;39:E1001-9.

13. Genant HK, Wu CY, van Kuijk C, Nevitt MC. Vertebral fracture assessment using a semiquantitative technique. J Bone Miner Res 1993;8:1137-48.

14. Tsuchiya A, Ikeda S, Ikegami N, et al. Estimating an EQ-5D population value set: the case of Japan. Health Econ 2002;11:341-53.

15. Scheer JK, Tang JA, Smith JS, et al. Cervical spine alignment, sagittal deformity, and clinical implications: a review. J Neurosurg Spine 2013;19:141-59.

16. Ames CP, Smith JS, Eastlack R, et al. Reliability assessment of a novel cervical spine deformity classification system. J Neurosurg Spine 2015;23:673-83.

17. Passias PG, Soroceanu A, Smith J, et al. Postoperative cervical deformity in 215 thoracolumbar patients with adult spinal deformity: prevalence, risk factors, and impact on patient-reported outcome and satisfaction at 2-year follow-up. Spine (Phila Pa 1976) 2015;40:283-91.

18. Banno T, Togawa D, Arima $\mathrm{H}$, et al. The cohort study for the determination of reference values for spinopelvic parameters (T1 pelvic angle and global tilt) in elderly volunteers. Eur Spine J 2016;25:3687-93.

19. Yoshida G, Yasuda T, Togawa D, et al. Craniopelvic alignment in elderly asymptomatic individuals: analysis of 671 cranial centers of gravity. Spine (Phila Pa 1976) 2014;39:1121-7.

20. Ames CP, Blondel B, Scheer JK, et al. Cervical radiographical alignment: comprehensive assessment techniques and potential importance in cervical myelopathy. Spine (Phila Pa 1976) 2013;38(22 Suppl 1):S149-60.

21. Siemionow K, An H, Masuda K, Andersson G, CsSzabo G. The effects of age, sex, ethnicity, and spinal level on the rate of intervertebral disc degeneration: a review of 1712 intervertebral discs. Spine (Phila Pa 1976) 2011;36:1333-9.

22. Yukawa Y, Kato F, Suda K, Yamagata M, Ueta T. Agerelated changes in osseous anatomy, alignment, and range of motion of the cervical spine. Part I: Radiographic data from over 1,200 asymptomatic subjects. Eur Spine J 2012;21:1492-8.

23. Protopsaltis TS, Scheer JK, Terran JS, et al. How the neck affects the back: changes in regional cervical sagittal alignment correlate to HRQOL improvement in adult thoracolumbar deformity patients at 2-year follow-up. J Neurosurg Spine 2015;23:153-8.

24. Park MS, Moon SH, Lee HM, et al. The effect of age on cervical sagittal alignment: normative data on 100 asymptomatic subjects. Spine (Phila Pa 1976) 2013;38:E458-63.

25. Boden SD, McCowin PR, Davis DO, Dina TS, Mark AS, Wiesel S. Abnormal magnetic-resonance scans of the cervical spine in asymptomatic subjects: a prospective investigation. J Bone Joint Surg Am 1990;72:1178-84.

26. Okada E, Matsumoto M, Ichihara D, et al. Does the sagittal alignment of the cervical spine have an impact on disk degeneration? Minimum 10-year follow-up of asymptomatic volunteers. Eur Spine J 2009;18:1644-51.

27. Vernon H, Mior S. The Neck Disability Index: a study of reliability and validity. J Manipulative Physiol Ther 1991;14:409-15. 